\title{
Contribuição do Monitor de Eventos no Diagnóstico de Sintomas
}

\author{
Cesar J. Grupi, Silvio A. Barbosa, Célia R. Sampaio, Paulo J. Moffa \\ São Paulo, SP
}

\begin{abstract}
Objetivo - Avaliar o poder de contribuição do monitor de eventos sintomáticos no esclarecimento de sintomas.

Métodos - Foram estudados 64 pacientes encaminhados para esclarecimento de sintomas e que já haviam sido submetidos à gravação com Holter. Foram monitorizados, durante 15 dias, com gravador com memória circular com capacidade de registrar uma derivação do ECG (CM5), antes e após ativado pelo paciente. Na vigência de sintomas, o paciente acionava um comando do gravador que provocava a retenção do sinal do ECG, que era, posteriormente, transmitido a uma central via telefone.
\end{abstract}

Resultados - Em dois pacientes não foi possível a realização completa da monitorização, nos restantes, sintomas que motivaram a indicação do exame foram: palpitações (67,7\%), tonturas (32,3\%), síncopes (29\%) e outros (30,6\%). Em 85,5\% dos pacientes houve relato de sintomas, sendo que em $62,2 \%$ houve registro de alterações eletrocardiográficas, relacionadas aos sintomas: taquicardia sinusal, 45,5\%; extra-sístoles, 30,3\%; taquiarritmia supraventricular, 21,2\%; taquicardia ventricular, 3\% e bloqueio atrioventricular, 3\%. A $1^{a}$ transmissão motivada por sintoma ocorreu: $35,5 \%$ no $1^{\circ}$ dia, $33,9 \%$ do $2^{\circ}$ ao $5^{\circ}, 12,9 \%$ do $6^{\circ}$ ao $10^{\circ}$ e $3,2 \%$ do $11^{\circ}$ ao $15^{\circ}$. Nos pacientes onde a gravação com Holter não permitiu esclarecimento, o gravador de eventos registrou sintomas em $35,5 \%$.

Conclusão - Trata-se de método bem aceito pelos pacientes e capaz de produzir aumento significativo no esclarecimento de sintomas em relação ao Holter.

Palavras-chave: gravador de eventos sintomáticos, arritmias cardíacas, monitorização cardíaca

\section{The Contribution of the Loop Memory ECG Recorder to the Diagnosis of Symptoms}

Purpose - To evaluate the contribution of the loop memory recorder in improving the diagnosis of symptoms related to cardiac arrhythmias.

Methods - The study population was 64 patients whose Holter monitorization was inconclusive as to the diagnosis of symptoms related to cardiac arrhythmias. Each wore a patient-activated ambulatory electrocardiogram device for 15 days. The ECG recordings during the occurrence of symptoms were sent to the ECG receiving center by phone.

Results - Two patients did not conclude the study. The majority of the patients ( $85.5 \%$ ) experienced symptoms in the fifteen days of monitorization, and in $62.2 \%$ of these patients electrocardiographic events were observed. The main symptoms experienced by these patients were: palpitation (67.7\%), dizziness (32.3\%), and syncope (29\%). Other symptoms like breast pain, fatigue, indisposition and dyspnea were also noted in $30.6 \%$ of the patients. The main electrocardiographic disturbances observed were: sinus tachycardia (45.5\%), isolated premature beats (30.3\%), supraventricular tachycardia $(21.2 \%)$, ventricular tachycardia (3\%) and third degree AV block (3\%). We also observed that the first symptomatic recording occurred mainly in the initial days of monitorization (69.4\% in the first 5 days). The percentage of diagnoses was $35.5 \%$ in patients whose Holter monitoring had been inconclusive.

Conclusion - The cardiac loop ECG recorder therefore achieved an important incremental diagnostic yield.

Key-words: loop ECG recorder, cardiac arrhythmias, cardiac monitorization

Arq Bras Cardiol, volume 70 (nº 5), 309-314, 1998

Instituto do Coração do Hospital das Clínicas - FMUSP

Correspondência: César Grupi - Incor - Av. Dr. Enéas C. Aguiar, 44 - 05403-000 - São Paulo, SP

Recebido para publicação em 13/11/97

Aceito em 4/3/98
A transmissão do eletrocardiograma (ECG) por radiofreqüência, proposta por Norman Holter em $1957^{1}$, foi utilizada, no final dos anos 60 e início dos anos 70, nos pacientes pós-infarto do miocárdio, logo após alta hospitalar, e em programas de reabilitação física. Posteriormente, surgi- 
ram os equipamentos para transmissão de ECG por telefone, utilizados inicialmente nas clínicas de avaliação de marcapassos com transmissão em tempo real ${ }^{2,3} \mathrm{e}$, hoje, com os sistemas de memória circular, também utilizados para o esclarecimento de sintomas, freqüentemente, relacionados a arritmias cardíacas, como palpitações, tonturas e síncopes. Sintomas que podem ser esclarecidos através da monitorização transtelefônica ${ }^{4-6}$, que também pode ser utilizada na avaliação da angina ${ }^{7}$, em acompanhamento de programas de reabilitação pós infarto do miocárdio ${ }^{8,9}$ e no ajuste de terapêutica anti-arrítmica ${ }^{10}$.

Este procedimento tem como sinônimos gravador de memória circular, monitor de eventos sintomáticos, sistema looping ou looper pela analogia com o sistema criado por Holter.

O monitor de eventos sintomáticos pelo fato de ser de fácil manipulação e de pequeno tamanho, pode ser utilizado por longo tempo e por isso torna-se vantajoso para o diagnóstico de sintomas de ocorrência esporádica.

Este sistema foi utilizado em pacientes encaminhados para tentativa de esclarecimento de sintomas, provavelmente, ligados às arritmias cardíacas. $\mathrm{O}$ objetivo do estudo foi: avaliar o tipo de sintoma que motivou a solicitação do exame, correlacionando-o com eventuais alterações eletrocardiográficas e estabelecer o período da monitorização que o paciente relatou, pela primeira vez, o sintoma que motivou o exame e compará-lo à monitorização com gravador de eventos e Holter de $24 \mathrm{~h}$, em relação ao benefício diagnóstico.

\section{Métodos}

Foram estudados 64 pacientes encaminhados por médicos do sistema público e privado para esclarecimento de sintomas, provavelmente, relacionados com as arritmias cardíacas. As idades variaram de 9 a 81 (média de 46) anos, sendo $39(61 \%)$ mulheres. Os pacientes foram esclarecidos quanto à realização do exame, da importância da sua participação no processo e treinados em relação à utilização do aparelho.

Os eletrodos e o preparo da pele foram os convencionais para o uso em monitorização com Holter. A monitorização se fez em posição de CM5, isto é, o eletrodo negativo colocado sobre o esterno ao nível do $2^{\circ}$ espaço intercostal direito e o eletrodo positivo sobre a $5^{\circ}$ costela ao nível da linha clavicular média, não havendo necessidade de eletrodo terra (fig. 1), permaneccndo instalados nas posições iniciais, exceto durante higiene pessoal, quando eram retirados e logo após reposicionados pelos próprios pacientes. Caso houvesse irritação da pele no local original, eram reposicionados em local próximo. O monitor permaneceu ligado, exceto no momento das transmissões. Por ser de pequeno tamanho, pôde ser transportado no bolso da camisa, por exemplo, permanecendo durante o sono ao lado do paciente.

Ao apresentarem os sintomas, os pacientes acionavam imediatamente o botão de registro situado na frente do monitor. No caso de síncope ou sintoma temporariamente incapacitante, o procedimento foi semelhante: após recobrar a capacidade funcional, o paciente acionava o dispositivo para gravação.

Os monitores utilizados foram de dois tipos: o $1^{\circ}$, não programável, com dois espaços de memória, cada um deles permitindo 60s do ECG antes e após o acionamento do botão; o $2^{\circ}$, programável, com memória total de 300 s, pode ser utilizada de acordo com as necessidades. Em pacientes com sintomas curtos e freqüentes, pode ser programado com

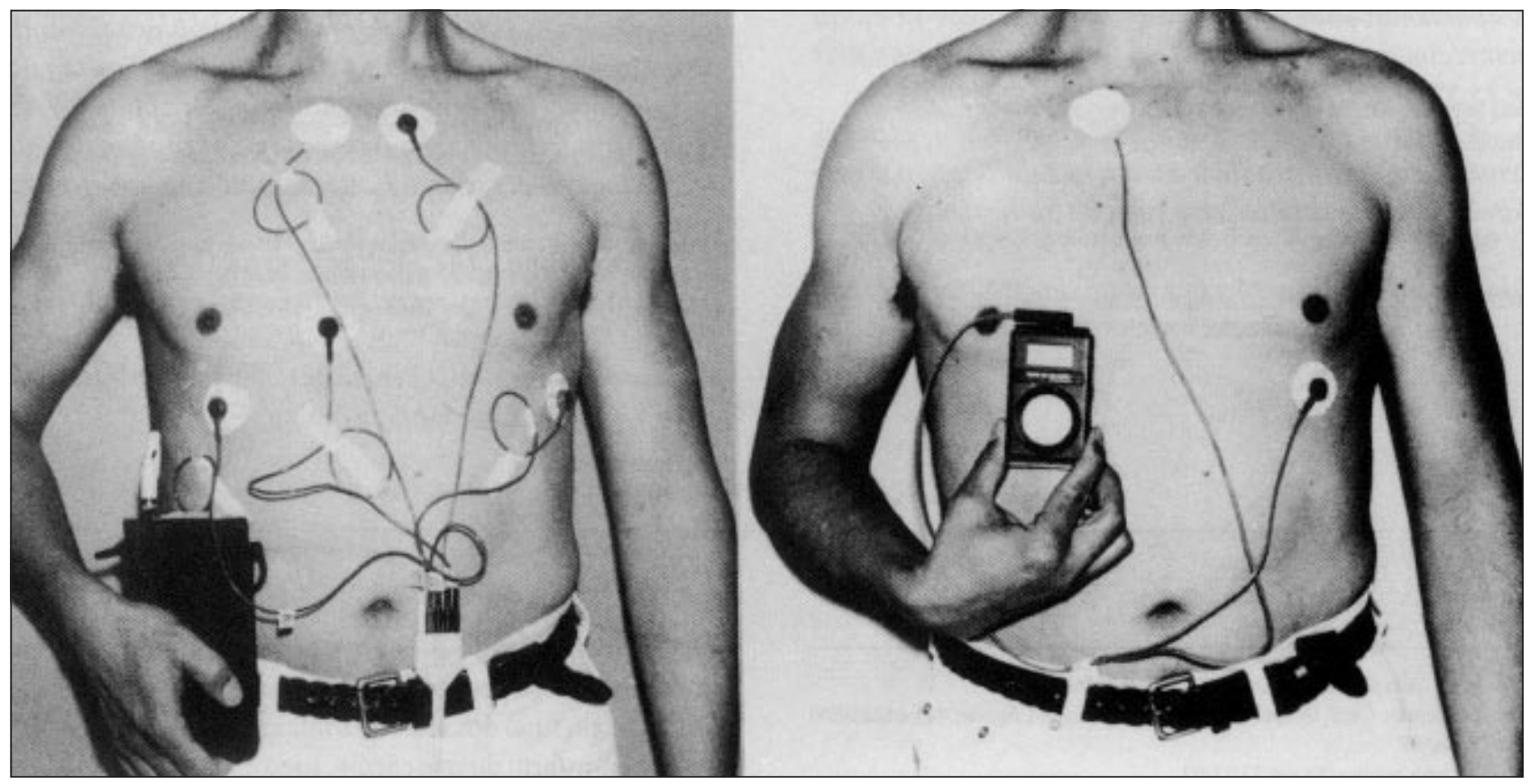

Fig. 1 - À esquerda, montagem para gravação no sistema Holter utilizando-se cinco eletrodos que permitem o registro de duas ou tres derivações simultâneas e um gravador com peso médio de 450g. À direita, mesmo paciente, com a instalação do sistema monitor de eventos sintomáticos, com dois eletrodos na posição CM5 e gravador que pesa 100g. 


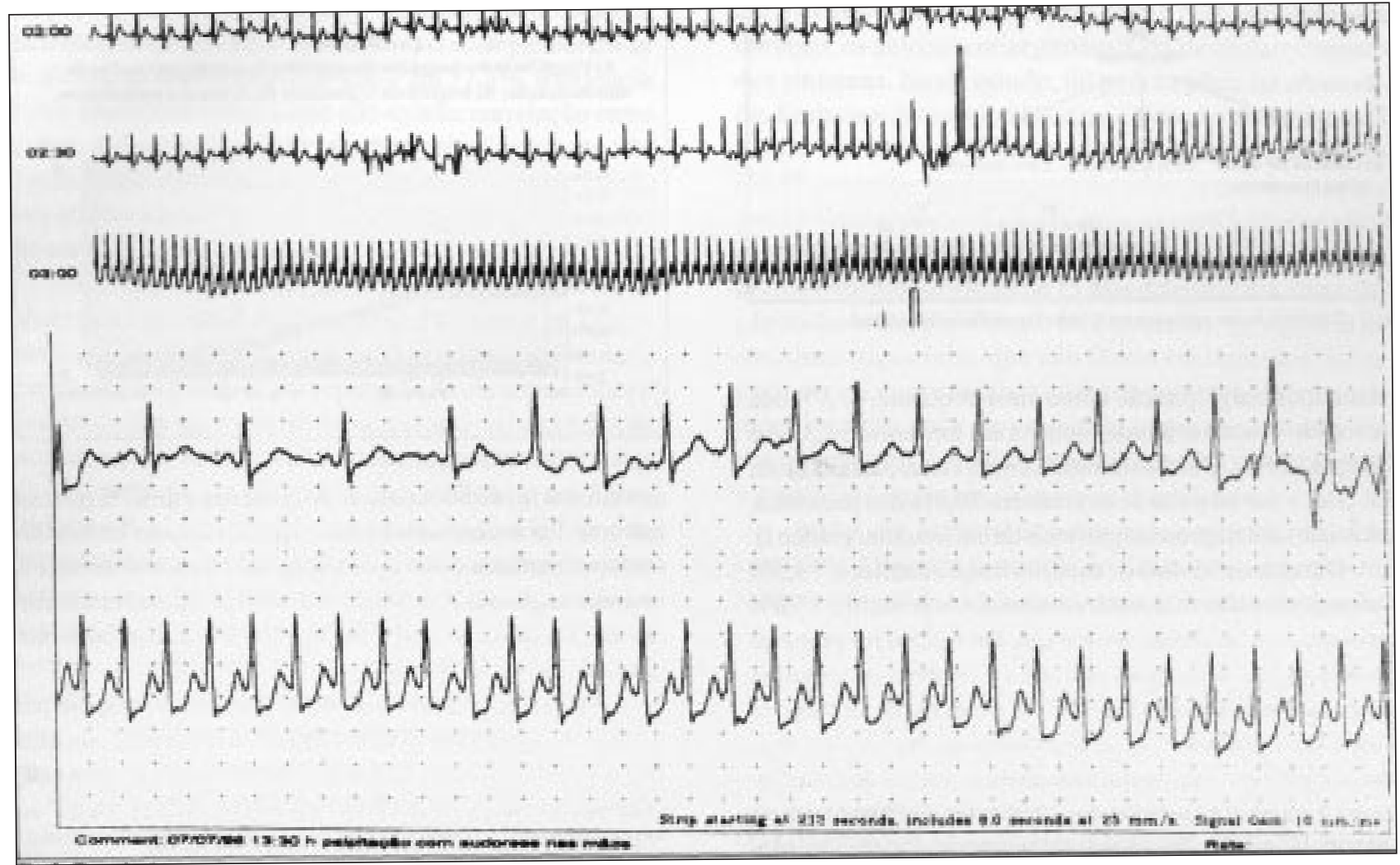

Fig. 2 - Traçado eletrocardiográfico recebido por transmissão transtelefônica. Nas 3 primeiras linhas a reprodução foi feita em 30 s/linha. Na $2^{\mathrm{a}}$ linha nota-se o início de taquicardia supra-ventricular, logo seguido de artefato que marca o momento em que o paciente acionou o gravador após sentir palpitação com sudorese nas mãos. Nos dois traçados inferiores, o ECG foi reproduzido a $25 \mathrm{~mm} / \mathrm{s}$. O $1^{\circ} \mathrm{com}$ ritmo sinusal com frequêencia cardíaca de $103 \mathrm{bpm}$ e o início da taquicardia; no $2^{\circ}$ traçado, registra-se taquicardia supra ventricular com freqüência de $240 \mathrm{bpm}$.

cinco espaços de memória, sendo 30s antes e após o acionamento do registro e, no outro extremo, pacientes com sintomas raros e temporariamente incapacitantes pode ser programado com um espaço de memória com 270s antes e 30s após o acionamento do registro, permitindo assim, a recuperação do ECG durante e até mesmo antes do início do sintoma. Os traçados eletrocardiográficos, assim gravados, foram posteriormente transmitidos via transtelefônica a uma central receptora para serem arquivados e avaliados a fim de ser estabelecido se houve alteração eletrocardiográfica e correlação com sintoma (fig. 2). O horário de recebimento das transmissões obedeceu ao de funcionamento do Serviço de Holter do INCOR, de 7:00 às 20:00h de segunda a sexta-feira e de 7:00 às 12:00h aos sábados. Durante o período noturno, nos fins de semana e feriados, os pacientes foram orientados para realizar os registros até preencher os espaços de memória dos seus monitores e transmiti-los no primeiro horário possível. As transmissões foram recebidas por técnicos treinados e sob supervisão médica constante. Ao ser identificada qualquer arritmia de risco ou de grande desconforto para o paciente, este foi imediatamente orientado para procurar o seu médico, sendo-lhe fornecido ou diretamente encaminhado ao médico, cópia do registro eletrocardiográfico.

Os pacientes, também, receberam um diário de atividades e sintomas onde anotavam as ocorrências no momento dos registros, semelhante ao que é feito no Holter, para de- pois serem confrontados com as informações fornecidas durante as transmissões.

O protocolo básico consistiu na monitorização contínua por 15 dias, com registro de pelo menos uma gravação ao dia, como teste, além de todas as gravações nas quais houvesse sintomas. A critério do médico assistente, a monitorização podia ser interrompida antes de concluído o período básico ou prolongada por novo período de observação.

O período de monitorização foi dividido em quatro períodos para análise do momento de ocorrência da $1^{\mathrm{a}}$ transmissão sintomática: $1^{\circ}$ dia, do $2^{\circ}$ ao $5^{\circ}$ dia, do $6^{\circ}$ ao $10^{\circ}$ dia e do $11^{\circ}$ ao $15^{\circ}$ dia.

Os resultados do monitor de eventos sintomáticos foram comparados com as gravações de Holter, quando disponíveis.

\section{Resultados}

Dois pacientes não conseguiram concluir o exame por dificuldade de manipulação do equipamento. As causas, presentes em ambos, foram: o equipamento não permanecia ligado e não foram capazes de acionar o botão de registro no momento dos sintomas, tiveram dificuldades na transmissão do ECG e não faziam anotação correta dos sintomas. Nenhum paciente interrompeu ou solicitou a interrupção do exame por desconforto relacionado aos eletrodos ou ao gravador.

A análise dos sintomas que motivaram a realização do 


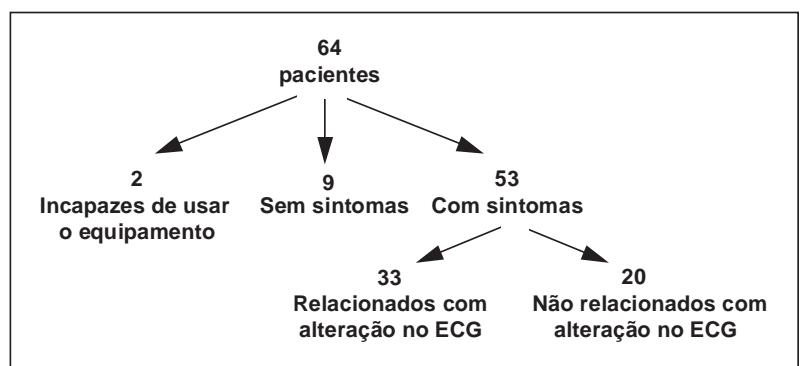

Fig. 3 - Distribuição dos pacientes em relação à ocorrência de sintomas.

exame aponta a palpitação como mais freqüente, $67,7 \%$ dos pacientes. Posteriormente, ocorreram tonturas $(32,3 \%) \mathrm{e}$ síncopes (29\%). Outros sintomas, como cansaço, falta de ar, mal estar e dor no peito ocorreram em $30,6 \%$ dos pacientes, sendo que vários apresentaram mais de um sintoma (gráfico I).

Durante os 15 dias de monitorização cardíaca, 14,5\% dos pacientes não relataram sintomas. Os restantes $85,5 \%$ apresentaram sintomas, sendo que em $62,2 \%$ foi possível correlacionar os sintomas com a presença de eventos eletrocardiográficos (fig. 3). As principais alterações eletrocardiográficas que se correlacionaram aos sintomas relatados foram: taquicardia sinusal em $45,5 \%$ dos pacientes; extra-sístoles isoladas em 30,3\%; taquiarritmia supraventricular em 21,2\%; taquicardia ventricular em 3\%; bloqueio atrioventricular de alto grau em $3 \%$ (gráfico II).

A percentagem de esclarecimento em relação ao tipo
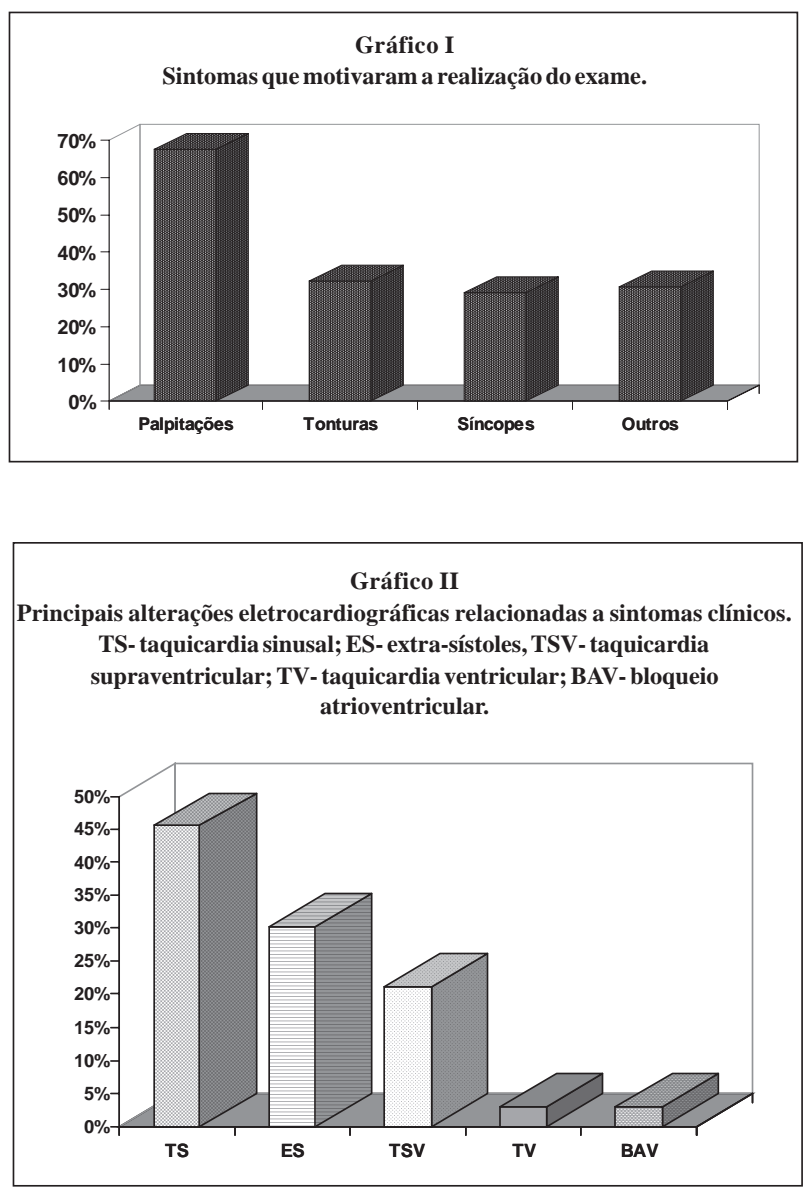

Gráfico III

A) Freqüência de chamados sintomáticos de acordo com os dias de monitorização; B) frequiência acumulada de chamados sintomáticos.

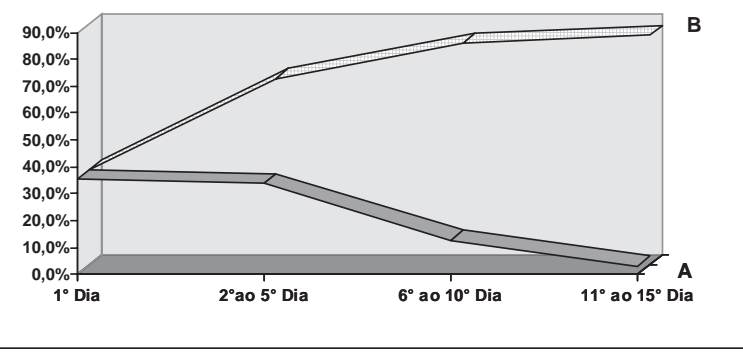

de sintoma foi de $96 \%$ para as palpitações e de $67 \%$ para as tonturas. Foram encaminhados 18 pacientes com história de síncope; nenhum deles apresentou este sintoma durante a monitorização e em um constatou-se taquicardia ventricular rápida durante a monitorização, evento potencialmente desencadeante de síncope.

A ocorrência da primeira manifestação do sintoma que motivou o exame, em relação aos períodos predeterminados foi: absoluta - $1^{\circ}$ dia $(35,5 \%) ; 2^{\circ}$ ao $5^{\circ}(33,9 \%) ; 6^{\circ}$ ao $10^{\circ}$ $(12,9 \%) ; 11^{\circ}$ ao $15^{\circ}(3,2 \%)$; cumulativa $-1^{\circ} \mathrm{dia}(35,5 \%) ; 2^{\circ}$ ao $5^{\circ}(69,4 \%) ; 6^{\circ}$ ao $10^{\circ}(82,3 \%) ; 11^{\circ}$ ao $15^{\circ}(85,5 \%)$

Embora todos os pacientes tivessem informado que já haviam se submetido a gravações de Holter, somente em 31 (48\%) deles conseguimos os traçados, sendo nestes possível a comparação com o registro do monitor de eventos sintomáticos. Em 11 (35,5\%) casos obteve-se a correlação do sintoma com alteração eletrocardiográfica.

\section{Discussão}

O registro eletrocardiográfico simultâneo ao sintoma presumivelmente de causa arrítmica é o padrão ouro para o seu diagnóstico. As grandes limitações para se atingir este objetivo são a freqüência, a duração e a incapacidade funcional dos sintomas. Porém, algumas condições devem ser observadas: se o sintoma é raro, porém, persistente, com ou sem incapacitação funcional, o paciente poderá ser levado a um serviço de emergência onde o registro do ECG convencional, isoladamente ou associado à derivação esofágica ou manobras de estimulação vagal, permitirá o diagnóstico. Se os sintomas têm frequiência em torno de uma vez ao dia, a monitorização com o sistema Holter é ideal. Entretanto, se os sintomas são ocasionais, uma vez por semana ou mais, sem ou com comprometimento funcional grave, é desconfortável e caro para o paciente estender-se a monitorização com Holter até a ocorrência do sintoma. Assim, a possibilidade de monitorização contínua do ECG que possa ser prolongada por vários dias com pouco desconforto para o paciente torna-se uma ferramenta fundamental. Neste estudo nenhum paciente interrompeu o exame por desconforto e somente dois não o terminaram por incapacidade de manuseio do equipamento.

Os resultados mostraram que a monitorização com 
Looper foi capaz de surpreender sintomas em $85,5 \%$ dos pacientes, sendo que $62,2 \%$, os sintomas acompanharam-se de alterações eletrocardiográficas e em $37,8 \%$, a ausência destas alterações indicou que não existiu correlação entre ambos. A documentação de que determinado sintoma não se relaciona a alterações eletrocardigráficas torna-se válida, pois orienta a busca de outras causas cardíacas ou mesmo não cardíacas para explicá-lo ${ }^{11}$.

É de se notar que esses pacientes referiram já terem se submetido à gravação com Holter de $24 \mathrm{~h}$, sem o esclarecimento exigido pelos seus médicos. Além disso, ao estudar o grupo onde foi possível a comparação direta entre Holter e monitor de eventos notou-se um acréscimo real de 35,5\% de pacientes que tiveram esclarecidos os seus sintomas. Estes achados estão de acordo com os de Grodman e $\operatorname{col}^{12}$ que, comparando a utilidade da monitorização com Holter e da transmissão transtelefônica para o esclarecimento de sintomas, encontraram nítida vantagem do segundo, sendo os dois igualmente capazes na identificação de arritmias assintomáticas. Os autores, entretanto, não fizeram referência sobre a capacidade de memória circular do sistema utilizado, o que poderia produzir resultados ainda mais favoráveis à transmissão transtelefônica.

O fato de eventos ocorrerem de forma repetitiva durante a monitorização prolongada, permite maior chance de correlação do que no Holter. Além disto, o registro doECG se faz simultaneamente à ocorrência do sintoma, não permitindo dúvida temporal, como freqüentemente ocorre na interpretação do Holter.

Embora, em muitos pacientes, o sintoma se correlacionasse com alterações que podem ser consideradas praticamente fisiológicas, como a taquicardia sinusal, ou arritmias sem importância clínica, como as extra-sístoles isoladas, em outros, foi possível o diagnóstico de arritmias com significado clínico relevante, como a taquicardia ventricular, bloqueios ou arritmias supra-ventriculares.

O tempo de monitorização necessário para se obter boa exatidão do método constitui motivo de considerações a respeito do custo-benefício do procedimento. Na monitorização com Holter a duração constitui fator crítico para identificação dos distúrbios do ritmo, sendo tanto mais efetiva quanto mais longa ${ }^{13}$. Durante a monitorização com o gravador de eventos sintomáticos, sendo possível o seu prolongamento indefinidamente, cumpre determinar sua duração ideal ${ }^{11}$.

A literatura mostra gráficos em que o número de transmissões sintomáticas obedece uma curva decrescente com a maioria delas ocorrendo nos primeiros dias de monitorização ${ }^{14}$. A observação destas curvas levou-nos a pro- por o uso do monitor de eventos por 15 dias, pois com esta duração, os autores conseguiram $87 \%$ de esclarecimento dos sintomas. Neste estudo, tal padrão pôde ser observado. Embora o protocolo limitasse a duração do exame a 15 dias, a maioria dos chamados telefônicos sintomáticos $(69,4 \%)$ ocorreu nos primeiros cinco dias de monitorização e por 15 dias foi possível obter sintomas em $85,5 \%$ dos pacientes. Portanto, segundo a curva do gráfico obtido, o seu prolongamento para mais de 15 dias determinaria uma relação custo-benefício discutível. Entretanto, na vigência de sintomas sugestivos, que não forem esclarecidos, ou na persistência de eventuais dúvidas diagnósticas, o tempo de monitorização pode ser estendido, o que se constituiu em outra vantagem do método, permitindo maior maleabilidade e propiciando maiores chances de diagnóstico.

A síncope, sintoma caracterizado por perda súbita de consciência seguida por perda do tônus muscular, embora sempre submetida a um extenso protocolo de investigação, permanece com freqüência sem diagnóstico, ultrapassando, por vezes, índices de até $50 \%$ dos pacientes ${ }^{15,16}$. O uso exclusivo da monitorização com Holter de $24 \mathrm{~h}$ mostra resultados com valores aquém de $10 \%{ }^{16,17,18}$. Entretanto, apesar das limitações conhecidas, o Holter de 24h ainda é o exame não invasivo mais utilizado na avaliação da síncope e também de sintomas relacionados a arritmias cardíacas. Neste estudo, o monitor de eventos sintomáticos utilizado por 15 dias conseguiu fazer o diagnóstico em somente um paciente além dos que já haviam se submetido ao Holter.

O sintoma que teve a maior percentagem de esclarecimento foi a palpitação (96\%). Este fato também se observa em outras publicações com o monitor de eventos ${ }^{19} \mathrm{e} e$ semelhante ao que se obtém com Holter. Isto é fácil de entender em função da frequiência de ocorrência do sintoma: quanto mais freqüente, mais fácil de ser surpreendido. Nos pacientes com síncope, como o intervalo entre os episódios geralmente é maior, o registro eletrocardiográfico simultâneo a um episódio sincopal é mais difícil de ser obtido, obrigando a monitorizações muito prolongadas. Nesses casos, a duração da monitorização tem importância fundamental, uma vez que, se fosse estendida, provavelmente poderia surpreender mais episódios de síncope ${ }^{6}$.

Concluímos que o gravador de eventos com a transmissão trans-telefônica do ECG constitui-se em método tecnicamente simples e muito bem tolerado pelos pacientes, podendo ser usado por longos períodos e sendo capaz de esclarecer o diagnóstico de sintomas que possam ter relação com as arritmias cardíacas, principalmente se estes sintomas tiverem uma ocorrência esporádica e que complementa outros métodos de diagnóstico cardiológico.

\section{Referências}

1. Holter NJ - Radioelectrocardiography - A new technique for cardiovascular studies. Ann Sci 1957; 65: 913-6.

2. Levine PA, Bellot PH, Bilitch M et al - Recommendations of the NASPE Policy Conference on Pacemaker Programmability and follow-up. PACE 1983; 6: 1222-32.
3. Rubin JW, Elison RG, Moore HV, Pai GP, Frank MJ, Killan HAW - Influence of telephone surveillance on pacemaker patient care. J Thorac Cardiovasc Surg 1980; 9: 218-26.

4. Ward DE, Camm AJ, Darby N - Assessment of the diagnostic value of 24 hour eletrocardiographic monitoring. Biotelem Patient Monitoring 1980; 7(2): 57-66. 
5. Linzer M, Pritchett ELC, Pontinen M, Mc Carthy E, Divine GW - Incremental diagnostic yield of loop eletrocardiographic recorders in unexplained syncope. Am J Cardiol 1990; 66: 214-9.

6. Linzer M, Prystowsky EN, Brunetti LL, Varia IM, German LD - Recurrent syncope of unknown origin diagnosed by ambulatory continuous loop ECG recording. Am Heart J 1988; 116: 1632-4.

7. Ginsburg R, Lamb IH, Schroeder JS, Harrison DC - Long-term transtelephonic electrocardiographic monitoring in the detection and evaluation of variant angina. Am Heart J 1981; 102: 196-201.

8. DeBusk RF, Haskell WL, Miller NH et al - Medically directed at home rehabilitation soon after clinically uncomplicated acute myocardial infarction: a new model of patient care. Am J Cardiol 1985; 55: 251-7.

9. Capone RJ, Stablein D, Visco J, Wagner G, Gorkin L, Follick MJ - The effects of a transtelephonic surveillance and prehospital emergency intervention system on the 1 year course following acute myocardial infarction. Am Heart J 1988; 116: 1606-15.

10. Hasin Y, David D, Rogel S - Transtelephone adjustment of antiarrhythmic therapy in ambulatory patients. Cardiology 1973; 63: 243-51.

11. Schmidt SB, Jain AC - Diagnostic utility of memory-equipped transtelephonic monitors. Am J Med Sci 1988; 296: 299-302.

12. Grodman RS, Capone RJ, Most AS - Arrhythmia surveillance by transtelephonic monitoring symptomatic ambulatory patients. Am Heart J 1979; 98: 459-64.

13. Kennedy HL, Chandra V, Sayther KL, Coralis DG -Effectiveness of increassing hours of continuos ambulatory electrocardiography in detecting maximal ventricular ectopy. Am J Cardiol 1978; 42: 925-30.

14. Reiffel JA, Schulhof E, Joseph B, Severance E, Wyndus P, McNamara A-Optimum duration of transtelephonic ECG monitoring when used for transient symptomatic event detection. J Electrocardiol 1991; 24: 165-8.

15. Silverstein MD, Singer DE, Mulley AG, Thibault GE, Barnett O - Patients with syncope admitted to medical intensive care units. JAMA 1982; 248: 1185-9.

16. Gendelman HE, Linzer M, Gabelman MM, Smoller S, Schever J - Syncope in a general hospital population. NY State Med J 1983; 83: 1161-5.

17. Luxon LM, Crowther A, Harrison MIG, Coltart J - Controlled study of eletrocardiographic monitoring in patients of transient neurologic symptoms. J Neurol Neurosurg Psychiatry 1980; 43: 37-41.

18. Gibson TC, Heitzman MR - Diagnostic efficacy of 24 hour eletrocardiographic monitoring for syncope. Am J Cardiol 1984; 53: 1013-7.

19. Kinlay S, Leitch J W, Neil A, Chapman B L, Hardy D B, Fletcher P J - Cardiac event recorders yield more diagnoses and are more cost-effective than 48-hour Holter monitoring in patients with palpitations. Ann Intern Med 1996; 124 $16-20$ 